\title{
Pulsed-Field Gel Electrophoresis
}

National Cancer Institute

\section{Source}

National Cancer Institute. Pulsed-Field Gel Electrophoresis. NCI Thesaurus. Code C17426.

Pulsed-field gel electrophoresis (PFGE) is a method used to separate high molecular weight linear DNA fragments that are greater than 50 kilobases (kb) in size. Fragments are separated by pulsing an electrical field across an agarose gel rather than applying the constant field used in conventional electrophoresis. 